\title{
A hypothesis of state covariance decorrelation effects to partial observability SLAM
}

\author{
H.Ahmad ${ }^{1}$, N.A Othman ${ }^{2}$, M.M Saari ${ }^{3}$, M.S Ramli ${ }^{4}$, M.M Mazlan ${ }^{5}$, T. Namerikawa ${ }^{6}$ \\ ${ }_{1,2,3,4}$ Faculty of Electrical \& Electronics Engineering, Universiti Malaysia Pahang, Malaysia \\ ${ }^{5}$ Electrical Department, Politeknik Sultan Hj Ahmad Shah, Kuantan, Malaysia \\ ${ }^{6}$ Namerikawa Laboratory, Department of System Design Engineering, Keio University, Japan
}

\begin{tabular}{l} 
Article Info \\
\hline Article history: \\
Received Sep 29, 2018 \\
Revised Nov 26, 2018 \\
Accepted Jan 28, 2019 \\
\hline Keywords: \\
Extended kalman filter \\
Matrix norm \\
Partial observability \\
SLAM
\end{tabular}

\begin{abstract}
This paper analyze the performance of partial observability in simultaneous localization and mapping(SLAM) problem. The study focuses mainly on the effect of having a decorrelation technique known as Covariance Inflation to the estimation. The matrix inversion will be the main element to be investigated through two conditions with respect to some defined environment namely as unstable partially observable SLAM and partially observable SLAM via matrix norm analysis. For assessment purposes, the Extended Kalman Filter estimation is referred as the estimator to understand how the conditions can influence the results. The simulation results depicted that, the matrix norm is able to determine the efficiency of estimation and is proportional to the uncertainties of the system.
\end{abstract}

Copyright $(2019$ Institute of Advanced Engineering and Science. All rights reserved.

\section{Corresponding Author:}

Hamzah Ahmad,

Faculty of Electrical \& Electronics Engineering,

Universiti Malaysia Pahang,

26600 Pekan, Pahang, Malaysia.

Email: hamzah@ump.edu.my

\section{INTRODUCTION}

Making an autonomous mobile robot is a great challenge as it requires a lot of unsolved issues. Researcher needs to consider various aspects such as the mobile robot structures, control algorithms, the surroundings area of observations, and dynamic situations. These factors have made the problem becomes difficult to be solved and requires more study considering those aspects. One of the navigation problem is known as Simultaneous Localization and Mapping(SLAM) problem that defines a condition where a mobile robot attempts to build a map consisting of itself and any observed landmarks concurrently while moving throuugh the environment[1]-[3]. SLAM has been a solution to various applications such as exploration, underwater navigation and for military purposes.

Generally there are three approaches in modeling the SLAM problem either by mathematical modelling, behavioral model or the probabilistic model. Each of the techniques has their own advantages that they are offering to researchers. Most notable performance has been recognized by using the probabilistic as there are many research applies Extended Kalman Filter to provide a sufficient information about the surrounding. The reason behind this is due to the probabilistics technique provides an efficient information with less computational cost and complexity in comparison with the other two methods mentioned above[1].

Among issues such as uncertainties[4]-[6], dynamic environments[7]-[9], complexity and computational cost[10]-[13], this paper attempts to study on reducing the computational cost by using a decorrelation technique on the state covariance. It is a problem that many approaches in SLAM are trying to solve by utilizing the information obtained from the state covariance. The state covariance is simply a representation of uncertainties that the mobile robot holds during its observation. The bigger the state 
covariance matrix size, more information about its surroundings are gathered[1]. Apart of those information, some of the landmarks locations are sometimes not required as only some unique landmarks are sufficient for the mobile robot references[2]. However, instead of neglecting landmarks, there is an idea to decorrelate some information obtained by the state covariance which in the end can reduce the computational cost as addressed by several authors e.g in [4], [11] and [12]. This will be the main perspective to be investigated in this paper.

A number of approaches have been proposed regarding to this matter for example, the covariance inflation method [4], and by adding pseudo state covariance to the predicted or updated state covariance [11]. The aim of those techniques are same i.e to make the computation becomes faster and at the same time can preserve a good estimation of both mobile robot and the landmarks. However, there are still exist some tradeoff such that if those methods have been applied, the error can become instantaneously high and result in erroneous estimation. Hence, this situation motivates further analysis and investigation in depth about why this is happening and requires any other possible technique for solution.

The analysis looks into the behavior of estimation by using the state covariance updates. The matrix inversion is this paper priority concerns to be assessed by evaluating the inversion of state covariance through a simple analysis of the matrix determinant. One of the possible way to assess the state covariance matrix is by examining the information matrix. The matrix norm of the information matrix is analyzed to understand its relation to the estimation behavior as it contains the magnitude of the information matrix. It was claimed that the decorrelation due to covariance inflation must be done properly to guarantee a reliable estimation [15]. Therefore, the calculation of state covariance matrix norm or the matrix magnitude can provide a picture on how to decorrelate the state covariance matrix without reducing the estimation performance. Further details are presented later in this paper.

The remaining parts of this paper are about the introduction to SLAM and its state covariance which will be presented in Section 2. A brief introduction to the Kalman Filter is also presented to provide a basic picture of how the system is working. This is followed by the analysis of the information matrix and matrix magnitude in Section3. Section 4 assess the proposition made in this paper and finally Section 5 concludes the paper.

\section{SLAM AND STATE COVARIANCE}

Two general steps are essential in determining a good localization and mapping by a mobile robot. The first step is known as process model or kinematic model that defines how the mobile robot moves in an environment. It then followed by the measurement or observation model where mobile robot taking relative measurements recursively at a period of time. These two steps can be mathematically presented as mentioned in the following subsections.

\subsection{Mathematical Formulation}

Consider a state $x_{k} \in \mathbb{R}^{(3+2 n)}$ which consists of mobile robot $\mathrm{x}, \mathrm{y}$ position and its heading angle with a number of $n$ landmarks marked with $\mathrm{x}, \mathrm{y}$ locations. The process model is represented by the following equation. The kinematic model of the system is represented by:

$$
x_{k+1}=f\left(x_{k}, u_{k}, \omega\right)
$$

where $u_{k}$ describes the control input that normally consists of the mobile robot velocity and angular acceleration. $\omega$ represents the noise occurred during mobile robot movements.

As mentioned previously, to achieve SLAM problem, the mobile robot needs to know its environment and therefore, the surroundings area must be observed. This is accomplished by using its sensors such as sonar sensor, vision sensor or the LRF sensor to measure the relative distance between mobile robot and any recognized landmarks during mobile robot observations. The measurement is calculated as follow.

$$
z_{k+1}=h\left(x_{k}, v\right)
$$

where $z_{k}$ describes the measurement matrix which consists of the relative distances and angles between mobile robot and landmarks.

\subsection{Kalman Filter and State Covariance}

Kalman Filter is generally build up based on two stages which are the prediction and update. Both of these stages are very important to be calculated to make recursive estimation of the mobile robot states and 
describing the condition of the area to be observed. Prediction stage is referring to the kinematic model of the mobile robot to infer the location of the mobile robot based on its movements. On the other hand, the update stage innovates the information obtained from the prediction through Kalman gain, to estimates the mobile robot and landmarks location at each sampled time.

The prediction stage holds the following equation.

$$
\hat{x}_{k+1}^{-}=\hat{x}_{k}+f\left(\hat{x}_{k}, u_{k}\right)
$$

where $\hat{x}_{k}$ is the predicted states with its associated state covariance matrix expressed by:

$$
\left.P_{k+1}^{-}=f P_{k}^{-} f^{T}+Q_{k} u_{k}\right)
$$

For above equation, $P_{k}^{-}$is the predicted state covariance with its associated noise, $Q_{k}$.

The information obtained in the prediction stage is then utilized to update the estimated state. The updated states $\hat{x}_{k+1}^{+}$becomes,

$$
\left.\hat{x}_{k+1}^{+}=\hat{x}_{k+1}^{-}+K\left(z_{k+1}-h\left(\hat{x}_{k+1}^{-}\right)\right) u_{k}\right)
$$

where $\mathrm{K}$ is the Kalman Gain.

$$
\left.K=P_{k+1}^{-} h^{T}\left(h P_{k+1}^{-} h^{T}+R_{k}\right)^{-1} u_{k}\right)
$$

where $R_{k}$ is the covariance of measurement error produced by the sensor. Together with the state updates is the updated state covariance shown as

$$
\left.P_{k+1}^{+}=(I-K h) P_{k+1}^{-} u_{k}\right)
$$

Information matrix is one of the available technique to analyze the condition of estimation. It is simply the inverse of the state covariance. Both calculation can be done for both prediction stage and updated stage. The information matrix at prediction stage yields

$$
\left(\Omega^{-}\right)_{k+1}^{-1}=Q_{k}^{-1}-Q_{k}^{-1} f\left(\left(\Omega^{+}\right)_{k}^{-1}+f^{T} Q_{k}^{-1} f\right)^{-1} f^{T} Q_{k}^{-1}
$$

For the updated stage, it becomes,

$$
\left(\Omega^{+}\right)_{k+1}^{-1}=P_{k+1}^{+}=\left(P_{k}^{-}\right)^{-1}+h^{T} R_{k}^{-1} h
$$

This is where the matrix inversion takes place which can consumed a lot of time when doing computation especially when the environment to be observed is bigger. Note that when the mobile robot observes more landmarks, the state size increases and consequently results in bigger matrix size for the state covariance. Note that it is desirable to design a system that has faster computation, as slower computation will not makes the SLAM problem becomes practical for real time applications. In matrix calculation especially when bigger matrix size is concerned, the calculation will be faster if the matrix to be inverted only has values on its diagonal elements. If the cross-diagonal elements are zero, then the calculation will be simpler. This is the point that motivates this research. To illustrate this, Figures 1 , and 2 provide a basic picture of the proposal of this research.

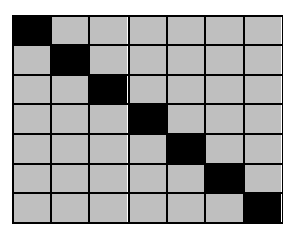

Figure 1. Full state covariance matrix (shaded area contains real number)

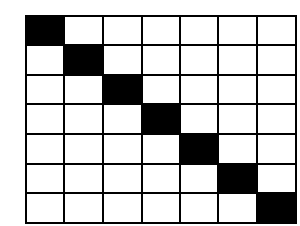

Figure 2. Decorrelated state covariance matrix (non-shaded area do not contains value) 


\section{INFORMATION MATRIX AND ITS MAGNITUDE}

This section investigates how the information matrix i.e the inverse of state covariance matrix behaves if some parts or elements in the matrix are modified to ease and makes faster computations. To begin with consider the following idea of a monobot system observing a landmark. Assume that there is a semidefinite matrix, $\mathrm{P}$ and $\mathrm{P} \geq 0$. Note also that, all elements in $\mathrm{P}$ is bigger than 0 .

$$
P=\left[\begin{array}{ll}
a & b \\
c & d
\end{array}\right]
$$

The inverse of $\mathrm{P}$ can be shown as follow.

$$
\Omega^{-1}=\left[\begin{array}{ll}
\alpha & \beta \\
\gamma & \delta
\end{array}\right]
$$

The inverse of matrix $\mathrm{P}$ is calculated based on normal matrix inverstion formula presented as follow.

$$
\Omega^{-1}=\left[\begin{array}{ll}
\alpha & \beta \\
\gamma & \delta
\end{array}\right]=\frac{1}{a d-b c}\left[\begin{array}{cc}
d & -b \\
-c & a
\end{array}\right]
$$

The calculation will be much more simpler whenever the element of $\mathrm{b}$ and $\mathrm{c}$ in $\mathrm{P}$ are equal to zero. In this case, those elements are called the cross-correlation of matrix $\mathrm{P}$. When this is happening, then the inversion becomes

$$
\Omega_{c}^{-1}=\frac{1}{a d}\left[\begin{array}{ll}
d & 0 \\
0 & a
\end{array}\right]=\left[\begin{array}{ll}
\frac{1}{a} & 0 \\
0 & \frac{1}{d}
\end{array}\right]
$$

In SLAM, the updated covariance cannot be decorrelate without sufficient consideration on how the elements are connected to each other. If those correlations are removed without proper modifications, then the estimation results exhibit erroneous results such that, the uncertainties suddenly becomes higher. By neglecting those correlations also means that, the mobile robot states do not has any confident in where it is located and becomes lost. Looking at different perspective, matrix $\mathrm{P}$ in equation above can also be represented by the following equation where some pseudo values are being added to it.

$$
\Omega^{-1}=\frac{1}{a d-(-b+b)(-c+c)}\left[\begin{array}{cc}
d & -b+b \\
-c+c & a
\end{array}\right]=\frac{1}{a d}\left[\begin{array}{ll}
d & 0 \\
0 & a
\end{array}\right]=\left[\begin{array}{cc}
\frac{1}{a} & 0 \\
0 & \frac{1}{d}
\end{array}\right]
$$

These two equations yield the same result. Such condition has been illustrated in the preceding research where they called it as unstable partially observable SLAM [5]. This condition cannot guarantee a good estimation if it is happening during estimation process.

Now the remaining matter to understand is to ensure that the state covariance features the same information even though in matrix decorrelation. One of the answer is by analyzing the matrix magnitude or matrix norm. Matrix norm demonstrates the matrix magnitude or in SLAM case, the information that mobile robot possess during its observations. If the information is modified from having a large information to smaller information, then the mobile robot is expected to has less information. Hence, the mobile robot holds less confidence about its whereabout.

The analysis will focus only on euclidean norm as it demonstrates similarly to the pythagorean length on describing how the mobile robot moves. Again, examine matrix $\mathrm{P}$ defined in 10. Remark that to guarantee a positive semidefinite matrix, the element of $a, d>0$. In addition, in SLAM, the state covariance matrix is symmetric. Hence its inverse holds the same properties i.e symmetric. By calculating the euclidean norm of the information matrix,

$$
\operatorname{norm}(\Omega)=\sqrt{\left[\frac{1}{a d-b c}\right]^{2}\left(d^{2}+b^{2}+c^{2}+a^{2}\right)}>0
$$

Note that the above equation yields a real number at all time. When element $b$, and $c$ are being decorrelated, then

$$
\operatorname{norm}\left(\Omega_{c}\right)=\sqrt{\left[\frac{1}{a}\right]^{2}+\left[\frac{1}{d}\right]^{2}}>0
$$


Comparing between these two conditions, clearly,

$$
\operatorname{norm}(\Omega)>\operatorname{norm}\left(\Omega_{c}\right)>0
$$

This criteria defines that the norms can be related directly to information obtained by the mobile robot. One more important thing discovered is that, the inverse of the information matrix can not exhibit infinite such that,

$$
\infty>\left[\frac{1}{a d-b c}\right]>0
$$

If the inverse of information matrix becomes infinite, then it is impossible to estimate the information obtained by the mobile robot. These two behaviors lead to below proposition and lemma.

The analysis now moved to the case of using Covariance Inflation method. Based on the theoretical descriptions, the updated state covariance becomes smaller than the previous estimation results[4]. Utilizing this information, the following are proposed.

Proposition : Mobile robot has less information about its surrounding if there is any decorrelation of its nondiagonal elements.

Proof : The proof can be easily obtained based on eqs.(10)-(12) for each time sequence and therefore omitted.

Lemma 1: Assume that an information matrix, $\Omega \in \mathbb{R}^{n \times n}$ is a semidefinite matrix and holds the following conditions.

$$
\Omega_{k+1} \geq \Omega_{k} \geq 0
$$

Proof : The proof can similarly be obtained from eqs.(10)-(18) and therefore omitted.

The information matrix holds a matrix norm higher than the decorrelated information matrix norm if the following are satisfied.

a) The inverse of information matrix do not exhibit infinite during mobile robot observations.

b) At least some of the non-diagonal elements of the state covariance matrix are non-zero.

\subsection{Numerical Example}

To assess the above proposed Lemma 1, the following examples are presented. The examples are divided into three different cases consisting of the system that do not posses any decorrelation, the one that has partial decorrelation and finally the one that has full decorrelation of the state covariance.

a) A case of the updated state covariance when the mobile robot is decorrelated to some of the landmarks. Suppose that

$$
P=\left[\begin{array}{ccc}
1 & 0.9 & 0 \\
0.9 & 1 & -0.2 \\
0 & -0.2 & 3
\end{array}\right]
$$

Calculating the norm and determinant of the above matrix gives,

$$
\operatorname{norm}(P)=3.0246, \text { determinant }(P)=0.53
$$

b) A case of the updated state covariance when the mobile robot is decorrelated to all landmarks. As shown in (19) is then being decorrelated about its non-diagonal element that then yields,

$$
P_{d}=\left[\begin{array}{lll}
1 & 0 & 0 \\
0 & 1 & 0 \\
0 & 0 & 3
\end{array}\right]
$$

If this happen, then the norm and determinant becomes,

$$
\operatorname{norm}\left(P_{d}\right)=3 \text {, determinant }\left(P_{d}\right)=3
$$

Clearly by observing the matrix norm, the second case yields smaller norm. This demonstrates a case when mobile robot uncertainties becomes higher as there is less information about its surrounding. This is also means that the robot do not has any reference landmarks located near to it. Smaller matrix norm leads to less information obtained by the mobile robot and could ended in erroneous results. 
c) Adding pseudo state covariance to the updated state covariance case resulting in $P_{s}$.

If the diagonal elements of the updated state covariance of (21) is added by a pseudo covariance, then the matrix norm becomes bigger than (22) such that $P_{s}>P>P_{d}$

This result is also explaining what has happened to the results presented by literatures [4], [11] as adding a pseudo state covariance can lead to erroneous estimation.

\section{SIMULATION ANALYSIS AND DISCUSSION}

To evaluate the performance of decorrelation for better view of what is happening to the estimation, simulation on certain conditions are presented. The simulation parameters are described in Table 1 for references. The simulation focuses on EKF for both gaussian noise and non-gaussian noise. The reason why to include these aspects is to observe the differences of EKF performance when decorrelation of state covariances are considered.

For the gaussian noise case, the following figures are obtained. The decorrelation may used either the one presented in [4] or [11]. In this research, the method presented in [4] is referred. Figure 3 described the performance of normal EKF in comparison to the EKF with partial decorrelation. The decorrelation did not makes the estimation faulty and preserved good results. The MSE assessment in Figure 4 shows that the EKF with partial decorrelation has better performance compared to the normal EKF. This is the case when 3 landmarks are being decorrelated. Even if the based on the proposed conditions stated in previous section shows that fewer information is available when decorrelation is in place, the mobile robot still preserved a good estimation results. One of the possible reason why this is happening can be expected due to the EKF inconsistency reported in various numbers of research. The estimation becomes too optimistic and lead to the illustrated results. This conditions lead to further investigation that will be organized in future.

Table 1. Simulation Parameters

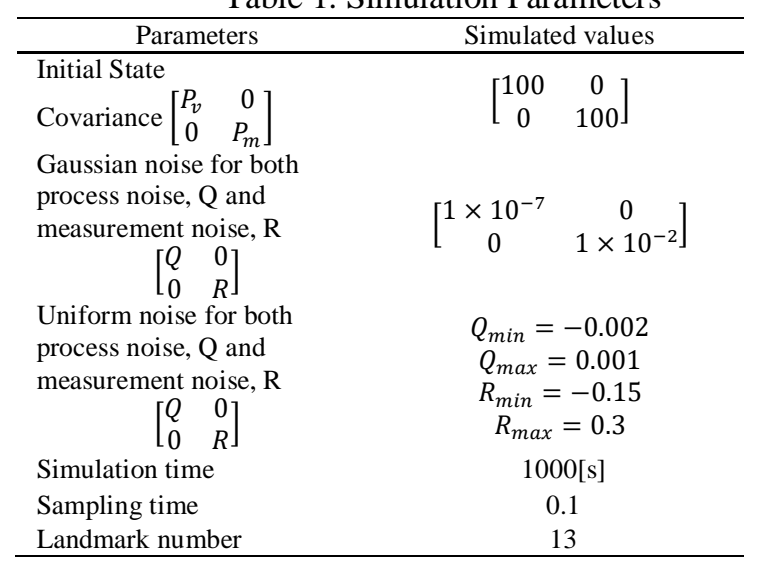

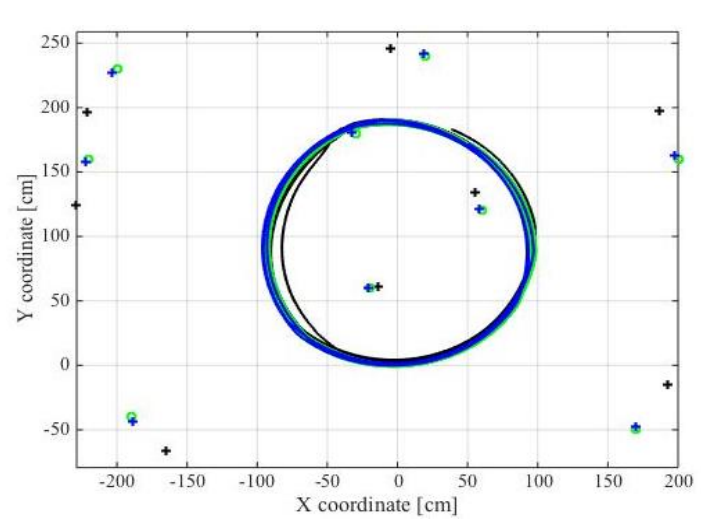

Figure 3. Estimation comparison between normal EKF with gaussian noise (black) and EKF with partial decorrelation (blue). The true path is mark as green
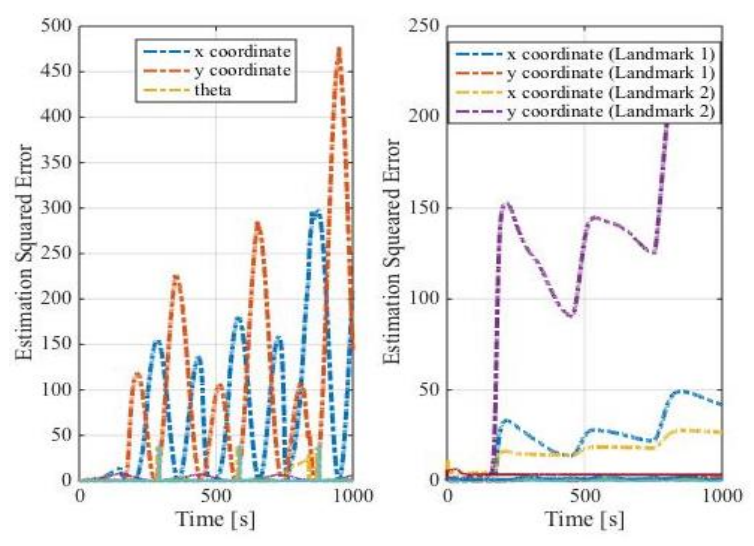

Figure 4. MSE results in gaussian noise between normal EKF(dash line) and EKF with partial decorrelation(solid line). 
Figure 5-7 are the case when only 3 landmarks are being decorrelated for non-gaussian noise case. Interestingly EKF with partial decorrelation still guarantees a good performance compared to the normal EKF.

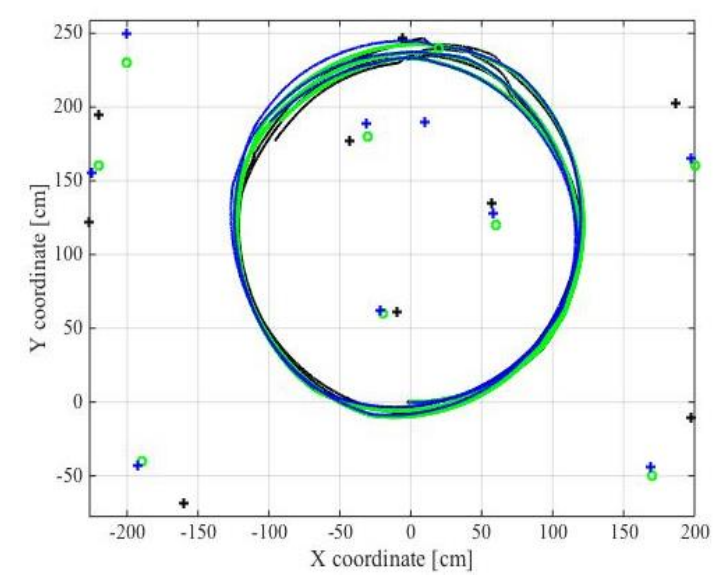

Figure 5. Estimation comparison between normal EKF with uniform noise (black) and EKF with partial decorrelation (blue). The true path is mark as green
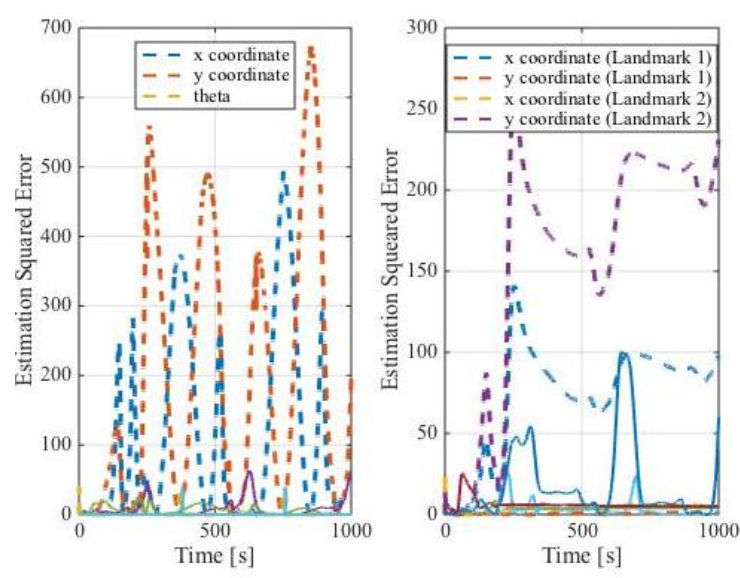

Figure 6. MSE results in uniform noise between normal EKF(dash line) and EKF with partial decorrelation(solid line)

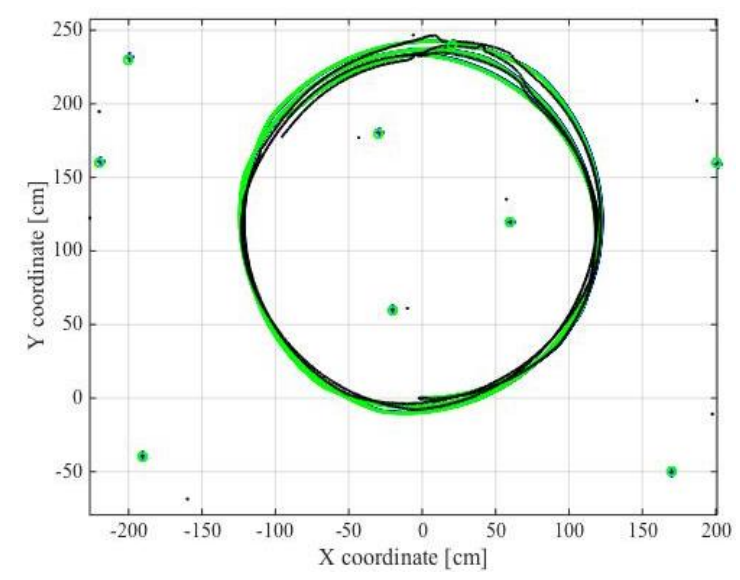

Figure 7. Estimation comparison between normal EKF with uniform noise (black) and EKF with full decorrelation (blue). The true path is mark as green.

The following Figures 7-8 are the results of decorrelating almost all landmarks. Surprisingly, even though almost all landmarks have been decorrelated to the mobile robot state covariance, the estimation can still preserved good results. This is due to the addition of the pseudo state covariance that able to compensate the decorrelation of the landmarks. Note that, the value of pseudo state covariance are selected based on certain readings of the non=diagonal state elements. Different to what previous research has suggested, the value of pseudo state covariance to add at each updated state covariance is changed as proposed earlier in (14). If the value of the pseudo state covariance is not correct or too big, then the state estimation become erroneous as shown in Figure 9. This is also called as unstable partially observable SLAM, where all non-diagonal elements in the updated state covariance are eliminated without considering their relations. 

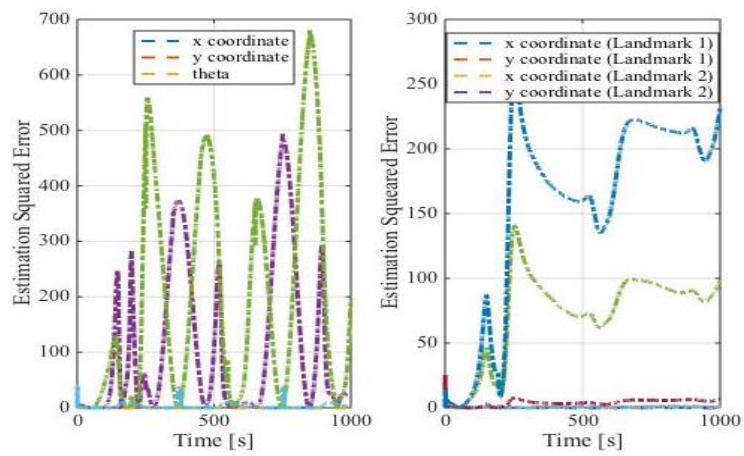

Figure 8. MSE results in uniform noise between normal EKF(dash line) and EKF with full decorrelation(solid line)

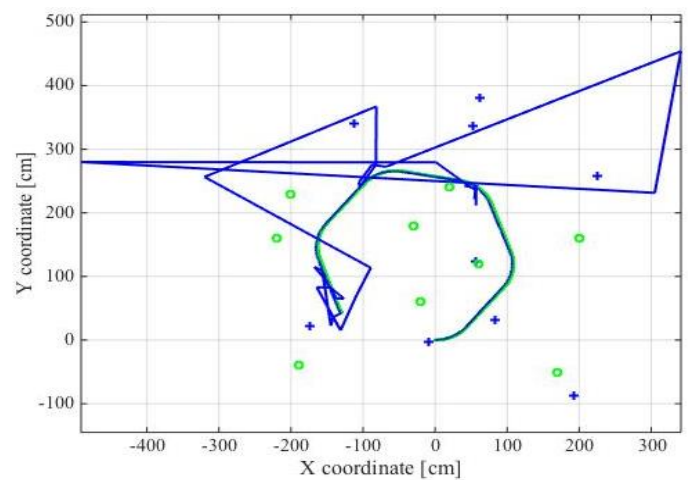

Figure 9. Estimation becomes erroneous when all landmarks are

\section{CONCLUSION}

This paper introduced the analysis of partially observability in SLAM problem by considering the effect of matrix inversion and decorrelation to the whole system. It was suggested that the information matrix can define the estimation performance if the information matrix is not suddenly become infinite and some of the elements in the state covariance is still holding a non-zero element. In addition, the results are consistent and agrees with what the preceding authors has claimed.

\section{ACKNOWLEDGEMENTS}

The authors would like to thank Ministry of Higher Education and Universiti Malaysia Pahang for supporting this research under RDU160145 and RDU160379.

\section{REFERENCES}

[1] S.Thrun, et al., "Probabilistic Robotic," 1st Edition, MIT Press, pp. 1-9, 2009.

[2] H.Durrant Whyte, et al., "Simultaneous Localization and Mapping: Part 1," IEEE Robotics \& Automation Magazine, Vol. 13(2), pp. 99-110, 2006.

[3] M. W. M. G.Disranayake, P. Newman, S.Clark, H. F. Durrant-Whyte, and M. Csorba. "A solution to the simultaneous localization and map building (SLAM) problem”, IEEE Trans. Robot. Automation. Vol. 17(3). pp. 229-241, 2001.

[4] S.Huang, M.W.M.G Dissayanake, "Convergence and Consistency Analysis for Extended Kalman Filter Based SLAM", IEEE Transactions on Robotics, Vol.23, no.5, pp. 1036-1049, 2007.

[5] J.Andrade-Cetto, et al., "The Effects of Partial Observability in SLAM," 2004 IEEE International Conference on Robotics \& Automation, Vol. 1, pp. 397-402, 2004.

[6] Guoquan P. Huang, et al., "On Filter Consistency of Discrete-Time nonlinear systems with partial-state measurements," in 2013 American Control Conference, ppp. 5468-5475, 2013.

[7] H.A Kadir, M.R Arshad, "Improved simultaneous localization and mapping (SLAM) algorithms for aerial vehicle under dynamic sea surface environment, 2016 IEEE Int. Conf. On Underwater System Technoogy: Theory and Applications(USYS), pp.61-66.

[8] F.S Vidal, A.O.P Barcelos, P.F F. Rosa,"SLAM solution based on particle filter with outliers filtering in dynamic environments", 2015 IEE 24th Int. Symp.on Industrial Electronics, pp. 644-649.

[9] P.Fritsche, B.Wagner, " Modeling structure and aerosal concentration with fused radar and LIDAR data in environment with changing visibility", 2017 IEEE/RSJ Int.Conf on Intelligent Robots and System, pp. 2685-2690.

[10] H.Ahmad, et al, "EKF based Slam with FIM Inflation", 2011 8th Asian Control Conference(ASCC2011), pp. 782-787, 2011.

[11] B.Noack, et al, "Treatment of Biased and Dependent Sensor Data in Graph-Based SLAM', 18th International Conference on Information Fusion, pp.1862-1867, 2015.

[12] T.Vidal-Valleja, et.al, "Conditions for Suboptimal Filter Stability in SLAM", 2004 IEEE/RSJ International Conference on Intelligent Robots and Systems(IROS), Vo1. pp. 27-32, 2004.

[13] A.Burguera, F Bonin-Font, G.Oliver, "Reducing the computational cost of underwater visual SLAM using dynamic adjustment of overlap detection”, 2014 IEEE Emerging Technology anf Factory Automation, pp.541-545.

[14] W.Burgard, O.Brock, C, Stachnicss, "Data association in O(n) for Divide and Conquer SLAM”, Robotics: Science and Systems III, MIT Press eBook Chapter, 2008.

[15] R.C.Smith and P.Cheereman."On the representation and estimation of spatial uncertainty", Robol. Res, Vol. 5(4), pp. 56-68. 1986. 


\section{BIOGRAPHIES OF AUTHORS}

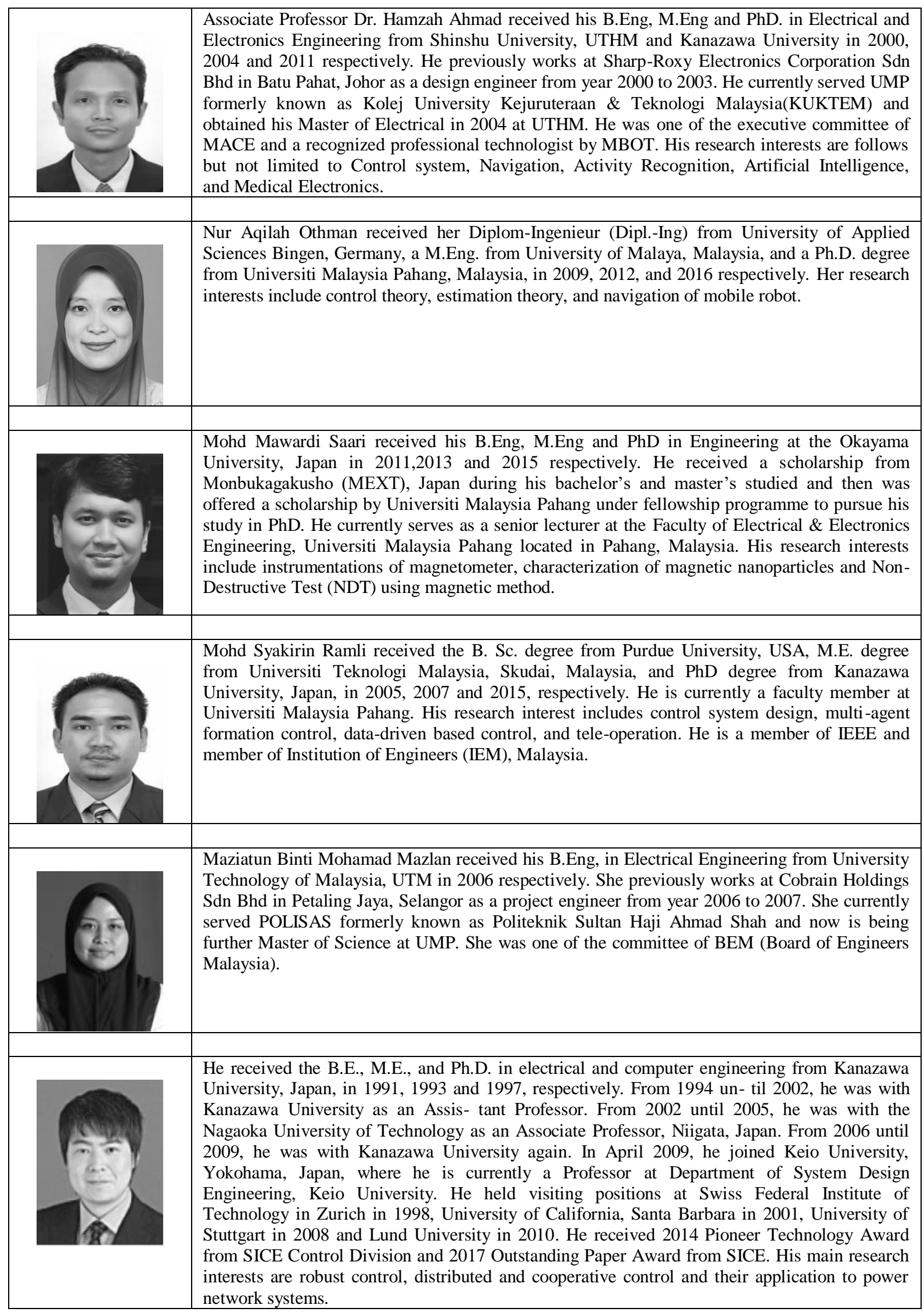

